\title{
The tensor reduction and master integrals of the two-loop massless crossed box ${ }^{*}$
}

\author{
Carlo Oleari \\ Department of Physics, University of Wisconsin, 1150 University Avenue, Madison WI 53706, U.S.A.
}

\begin{abstract}
We briefly discuss an algorithm for the tensor reduction of the two-loop massless crossed boxes, with light-like external legs, and the computation of the relative master integrals.
\end{abstract}

\section{INTRODUCTION}

The level reached nowadays by the precision measurements in high-energy scattering experiments demands the knowledge of next-to-next-to-leading theoretical amplitudes for $2 \rightarrow 2$ scattering processes.

Very recent results for two-loop scattering amplitudes for massless particle have already appeared in the literature: the maximal-helicity-violating two-loop amplitude for $g g \rightarrow g g$ [1], $e^{+} e^{-} \rightarrow \mu^{+} \mu^{-}$and $e^{+} e^{-} \rightarrow e^{-} e^{+}$[2], $q \bar{q} \rightarrow q^{\prime} \bar{q}^{\prime}[3]$ and $q \bar{q} \rightarrow q \bar{q}[4]$.

In dealing with these two-loop scattering amplitudes we have to face the problem of the tensor reduction of planar [5] and crossed double boxes [6], plus a plethora of simpler topologies [7,8], and the computation of the relative master integrals $[9,10]$.

\section{NOTATION}

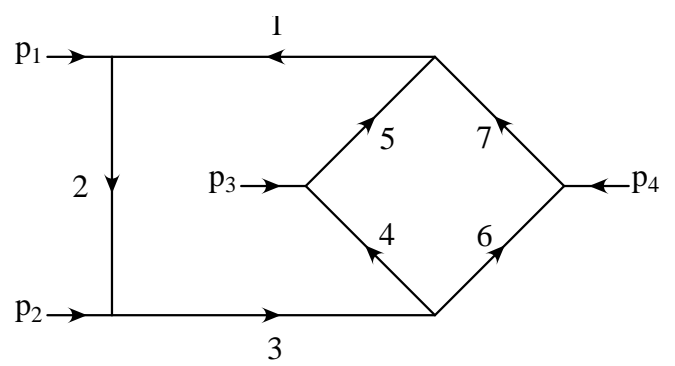

FIGURE 1. The generic two-loop crossed box.

* Talk given at ACAT 2000, Fermilab, Batavia, IL, October 16-20, 2000. To be published in Advanced Computing and Analysis Techniques in Physics Research, edited by: P.C. Bhat and M. Kasemann.
We denote the generic two-loop tensor crossed (or non-planar) four-point function in $D$ dimensions of Fig. 1 with seven propagators $A_{i}$ raised to arbitrary powers $v_{i}$ as

$$
\begin{aligned}
& \operatorname{Xbox}^{D}\left(v_{1} \ldots v_{7} ; s, t\right)\left[1 ; k^{\mu} ; l^{\mu} ; k^{\mu} k^{\nu} ; k^{\mu} l^{\nu} ; \ldots\right] \\
& =\int \frac{d^{D} k}{i \pi^{D / 2}} \int \frac{d^{D} l}{i \pi^{D / 2}} \frac{\left[1 ; k^{\mu} ; l^{\mu} ; k^{\mu} k^{\nu} ; k^{\mu} l^{\nu} ; \ldots\right]}{A_{1}^{v_{1}} A_{2}^{v_{2}} A_{3}^{v_{3}} A_{4}^{v_{4}} A_{5}^{v_{5}} A_{6}^{v_{6}} A_{7}^{v_{7}}},
\end{aligned}
$$

where the propagators are

$$
\begin{array}{ll}
A_{1}=\left(k+l+p_{34}\right)^{2}+i 0, & A_{2}=\left(k+l+p_{134}\right)^{2}+i 0, \\
A_{3}=(k+l)^{2}+i 0, & A_{4}=l^{2}+i 0, \\
A_{5}=\left(l+p_{3}\right)^{2}+i 0, & A_{6}=k^{2}+i 0, \\
A_{7}=\left(k+p_{4}\right)^{2}+i 0 . &
\end{array}
$$

The external momenta $p_{j}$ are in-going and light-like, $p_{j}^{2}=0, j=1 \ldots 4$, so that the only momentum scales are the usual Mandelstam variables $s=\left(p_{1}+p_{2}\right)^{2}$ and $t=\left(p_{2}+p_{3}\right)^{2}$, together with $u=-s-t$. For ease of notation, we define $p_{i j}=p_{i}+p_{j}$ and $p_{i j k}=p_{i}+p_{j}+p_{k}$. In the square brackets we keep trace of the tensor structure that may be present in the numerator.

\section{TENSOR REDUCTION}

As it is well known, tensor integrals can be related to combinations of scalar integrals with higher powers of propagators and/or different values of $D[7,11]$. This is quite straightforward to see if we rewrite the Feynman integral introducing the Schwinger parameters, diagonalizing the exponent of the out-coming integral and integrating out the loop momenta $[6,7]$.

The task to compute tensor integrals is then moved to the computation of scalar integrals with

- higher powers of the propagators

- in higher dimensions. 
From the Schwinger representation of Feynman integrals it easy to see that integrals in $D$ dimensions can be connected with integrals in $D+2$ dimensions (dimensionalshift) $[5,6]$.

In this way, tensor integrals can be directly connected to scalar integrals with higher powers of the propagators in $D=4-2 \varepsilon$ dimensions.

\section{THE SCALAR CROSSED-BOX REDUCTION}

The strategy to reduce the generic scalar integral to a linear combination of known ones is based on recurrence identities that relate scalar integrals with different powers of propagators. Some of these identities can be obtained using the integration-by-parts method [12] and exploiting the Lorentz invariance of the Feynman diagram [13].

Following the reduction procedure detailed in Ref. [6], any scalar crossed box with arbitrary powers of the propagators can be written as a linear combination of the following integrals, that, therefore, are called master integrals:

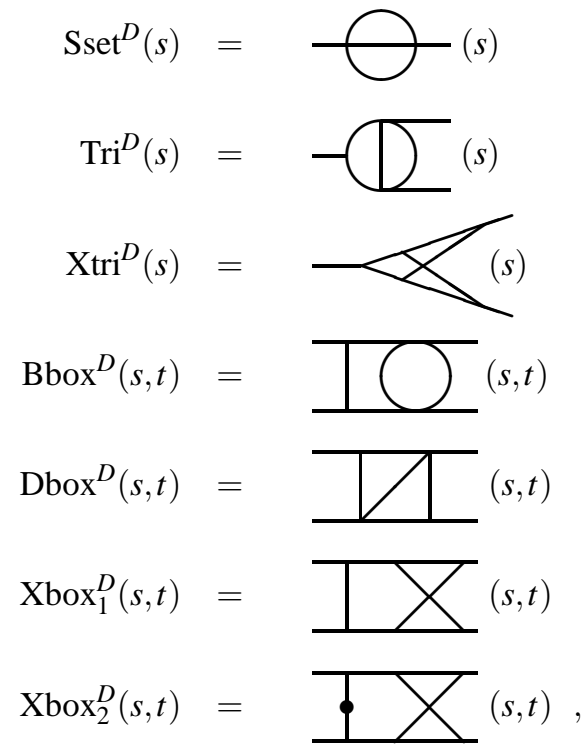

where all the propagators have powers one except for the propagator with the blob, that has power two.

\section{DIFFERENTIAL EQUATIONS FOR THE TWO MASTER INTEGRALS}

The analytic expansion in $\varepsilon=(4-D) / 2$ for the first master cross box $\operatorname{Xbox}_{1}^{D}(s, t)$ was computed in Ref. [10]. We can obtain the analytic form for the second one by writing the derivative of $\operatorname{Xbox}_{1}^{D}(s, t)$ with respect to one of the two independent physical scales (that we choose to be $t$ ), as a combination of master integrals, and solving the equation for $\operatorname{Xbox}_{2}^{D}(s, t)$. Moreover we can verify the correctness of both the expressions of $\operatorname{Xbox}_{1}^{D}(s, t)$ and $\operatorname{Xbox}_{2}^{D}(s, t)$, by deriving an analogous differential equation for $\mathrm{Xbox}_{2}^{D}$, and checking that the obtained identity is satisfied.

Starting from the Schwinger representation of the generic crossed box, we can differentiate with respect to $t$, and set the values of $v_{i}$ to reproduce the two master integrals

$$
\begin{aligned}
\frac{\partial}{\partial t} \operatorname{Xbox}_{1}^{D}(s, t)= & \operatorname{Xbox}^{D+2}(1,2,1,2,1,1,2 ; s, t) \\
& -\operatorname{Xbox}^{D+2}(1,2,1,1,2,2,1 ; s, t), \\
\frac{\partial}{\partial t} \operatorname{Xbox}_{2}^{D}(s, t)= & 2 \operatorname{Xbox}^{D+2}(1,3,1,2,1,1,2 ; s, t) \\
& -2 \operatorname{Xbox}^{D+2}(1,3,1,1,2,2,1 ; s, t) .
\end{aligned}
$$

Applying the reduction formalism for the scalar integrals and the dimensional-shift, we can rewrite the right-hand sides of the system as a combination of the two master crossed boxes plus other master integrals of simpler topologies, obtained by pinching one or more of the propagators of the crossed box,

$$
\begin{gathered}
\frac{\partial}{\partial t} \operatorname{Xbox}_{1}^{D}(s, t)=\frac{1}{t-u}\left[\frac{(D-4) s^{2}-4 t u}{2 t u} \operatorname{Xbox}_{1}^{D}(s, t)\right. \\
\left.-\frac{(D-6) s}{2(D-5)} \operatorname{Xbox}_{2}^{D}(s, t)+\text { pinchings }\right] \\
\frac{\partial}{\partial t} \operatorname{Xbox}_{2}^{D}(s, t)=\frac{1}{t-u}\left[\frac{2(D-5)^{2} s}{t u} \operatorname{Xbox}_{1}^{D}(s, t)\right. \\
-\frac{(D-6)\left(u^{2}+t^{2}\right)}{t u} \operatorname{Xbox}_{2}^{D}(s, t)+\operatorname{pinchings}^{2}
\end{gathered}
$$

Inserting the $\varepsilon$ expansion of $\operatorname{Xbox}_{1}^{D}(s, t)$ computed in Ref. [10] and the $\varepsilon$ expansions of the sub-topologies listed in Refs. [5, 7, 14, 15] into Eq. (1), and solving it with respect to $\operatorname{Xbox}_{2}^{D}(s, t)$, we obtain , in the physical region $s>0, t, u<0$,

$$
\begin{array}{r}
\operatorname{Xbox}_{2}^{D}(s, t)=\Gamma^{2}(1+\varepsilon)\left\{\frac{G_{1}(t, u)}{s^{3} t}+\frac{G_{2}(t, u)}{s^{2} t^{2}}+\right. \\
\left.\frac{G_{1}(u, t)}{s^{3} u}+\frac{G_{2}(u, t)}{s^{2} u^{2}}\right\},
\end{array}
$$

where

$$
\begin{aligned}
& G_{1}(t, u)=s^{-2 \varepsilon}\left\{\frac{6}{\varepsilon^{3}}+\frac{1}{\varepsilon^{2}}(32-6 T-6 U)\right. \\
& +\frac{1}{\varepsilon}\left(1-12 \pi^{2}-24 T+T^{2}-24 U+16 T U+U^{2}\right)
\end{aligned}
$$




$$
\begin{aligned}
& -43-18 T+13 T^{2}+\frac{8}{3} T^{3}-18 U+16 T U \\
& +11 T^{2} U+13 U^{2}-20 T U^{2}+\frac{8}{3} U^{3}+\pi^{2}(17 T \\
& \left.+17 U-\frac{112}{3}\right)-122 \zeta(3)+62 T \operatorname{Li}_{2}\left(-\frac{t}{s}\right) \\
& -62 \mathrm{Li}_{3}\left(-\frac{t}{s}\right)+62 \mathrm{~S}_{1,2}\left(-\frac{t}{s}\right) \\
& +i \pi\left[\frac{1}{\varepsilon}(16+6 T+6 U)-34-9 \pi^{2}-6 T-10 T^{2}\right. \\
& \left.\left.-6 U+14 T U-10 U^{2}\right]\right\} \\
& G_{2}(t, u)=s^{-2 \varepsilon}\left\{-\frac{2}{\varepsilon^{4}}+\frac{1}{\varepsilon^{3}}\left(-8+\frac{5}{2} T+\frac{7}{2} U\right)\right. \\
& +\frac{1}{\varepsilon^{2}}\left(-\frac{29}{2}-\frac{5}{12} \pi^{2}+7 T-T^{2}+20 U-4 T U\right. \\
& \left.-U^{2}\right)+\frac{1}{\varepsilon}\left[-\frac{1}{2}+17 T+2 T^{2}-\frac{T^{3}}{3}+\frac{\pi^{2}}{6}(14\right. \\
& +5 T-29 U)+13 U-28 T U-4 U^{2}+3 T U^{2} \\
& -U^{3}+\frac{19}{2} \zeta(3)-2 T \mathrm{Li}_{2}\left(-\frac{t}{s}\right)+2 \mathrm{Li}_{3}\left(-\frac{t}{s}\right) \\
& \left.-2 \mathrm{~S}_{1,2}\left(-\frac{t}{s}\right)\right]+\frac{37}{2}+\frac{37}{40} \pi^{4}+7 T-5 T^{2} \\
& -\frac{22}{3} T^{3}+\frac{2}{3} T^{4}+5 U-20 T U+\frac{8}{3} T^{3} U-2 U^{2} \\
& +24 T U^{2}-T^{2} U^{2}-8 U^{3}-\frac{4}{3} T U^{3}+\frac{4}{3} U^{4} \\
& +\frac{\pi^{2}}{6}\left(79-22 T-5 T^{2}-200 U+76 T U+25 U^{2}\right) \\
& +(68-13 T-33 U) \zeta(3)+\left(10 \pi^{2}-32 T+17 T^{2}\right. \\
& +12 T U) \mathrm{Li}_{2}\left(-\frac{t}{s}\right)-36 \mathrm{~S}_{2,2}\left(-\frac{t}{s}\right) \\
& +(28 T-6 U-32) \mathrm{S}_{1,2}\left(-\frac{t}{s}\right)-26 \mathrm{~S}_{1,3}\left(-\frac{t}{s}\right) \\
& +(32-60 T-12 U) \mathrm{Li}_{3}\left(-\frac{t}{s}\right)+86 \mathrm{Li}_{4}\left(-\frac{t}{s}\right) \\
& +i \pi\left[\frac{2}{\varepsilon^{3}}+\frac{1}{\varepsilon^{2}}(11-T+U)+\frac{1}{\varepsilon}\left(1-\frac{31}{6} \pi^{2}-10 T\right.\right. \\
& \left.-2 T^{2}+4 U-2 T U-2 U^{2}\right)+11+4 T-2 T^{2} \\
& +\frac{10}{3} T^{3}+\frac{\pi^{2}}{3}(-65+28 T-U)+2 U-8 T U \\
& -8 U^{2}+2 U^{3}-89 \zeta(3)+(14 T+18 U) \mathrm{Li}_{2}\left(-\frac{t}{s}\right) \\
& \left.\left.-32 \mathrm{Li}_{3}\left(-\frac{t}{s}\right)+44 \mathrm{~S}_{1,2}\left(-\frac{t}{s}\right)\right]\right\} \text {, }
\end{aligned}
$$

$T=\log (-t / s), \quad U=\log (-u / s)$, and where we used Nielsen's generalized polylogarithms $\mathrm{S}_{n, p}$ defined by

$$
\begin{aligned}
\mathrm{S}_{n, p}(x)= & \frac{(-1)^{n+p-1}}{(n-1) ! p !} \int_{0}^{1} \mathrm{~d} t \frac{\log ^{n-1}(t) \log ^{p}(1-x t)}{t} \\
& n, p \geq 1, \quad x \leq 1
\end{aligned}
$$

Expressions for $\operatorname{Xbox}_{2}^{D}(s, t)$ in the other two kinematic regions, $t>0, s, u<0$ and $u>0, s, t<0$ can be easily obtained through the analytic continuation of the polylogarithms and the logarithms.

It is a strong check of the whole formalism that inserting the analytic expansion of $\operatorname{Xbox}_{1}^{D}(s, t)$ and the derived expression of $\operatorname{Xbox}_{2}^{D}(s, t)$ into Eq. (2), we obtain an equality identically satisfied.

Acknowledgements. This work has been done in collaboration with C. Anastasiou, T. Gehrmann, E. Remiddi and J. B. Tausk.

\section{REFERENCES}

1. Z. Bern, L. Dixon and D.A. Kosower, JHEP 0001 (2000) 027, hep-ph/0001001.

2. Z. Bern, L. Dixon and A. Ghinculov, hep-ph/0010075.

3. C. Anastasiou, E.W.N. Glover, C. Oleari and M.E. TejedaYeomans, hep-ph/0010212.

4. C. Anastasiou, E.W.N. Glover, C. Oleari and M.E. TejedaYeomans, hep-ph/0011095.

5. V.A. Smirnov and O.L. Veretin, Nucl. Phys. $\mathbf{5 5 6 6}$ (2000) 469.

6. C. Anastasiou, T. Gehrmann, C. Oleari, E. Remiddi and J.B. Tausk, Nucl. Phys. B580 (2000) 577, hep-ph/0003261.

7. C. Anastasiou, E.W.N. Glover and C. Oleari, Nucl. Phys. B575 (2000) 416, Erratum-ibid $\mathbf{5 5 8 5}$ (2000) 763, hep-ph/9912251.

8. C. Anastasiou, E.W.N. Glover and C. Oleari, Nucl. Phys. B565 (2000) 445.

9. V.A. Smirnov, Phys. Lett. B460 (1999) 397.

10. J.B. Tausk, Phys. Lett. B469 (1999) 225.

11. O.V. Tarasov, Phys. Rev. 054 (1996) 6479, Nucl. Phys. B502 (1997) 455.

12. F.V. Tkachov, Phys. Lett. 100B (1981) 65; K.G. Chetyrkin and F.V. Tkachov, Nucl. Phys. $B 192$ (1981) 159.

13. T. Gehrmann and E. Remiddi, Nucl. Phys. B580 (2000) 485, hep-ph/9912329.

14. R.J. Gonsalves, Phys. Rev. D28 (1983) 1542.

15. G. Kramer and B. Lampe, J. Math. Phys. 28 (1987) 945. 